\title{
FICTITIOUS-DOMAIN SIMULATION OF SOLID-LIQUID FLOW WITH "SUBGRID" LUBRICATION FORCE CORRECTION; A SPHERE FALLING ONTO A PLANE SURFACE
}

\author{
Y NGUYEN ${ }^{1}$, John WELLS $^{2 *}$ and Hung TRUONG ${ }^{1 *}$ \\ Dept. of Civil \& Environmental Engineering, Ritsumeikan University \\ (Noji Higashi 1-1-1 Kusatsu, Shiga, 525-8577, Japan) \\ ${ }^{1}$ Graduate student, ${ }^{2}$ Associate Professor, * Member of JSCE
}

\begin{abstract}
A simple correction model for "subgrid" lubrication force is proposed for fictitious-domain simulation of solid-liquid flow employing the Variable-density Implicit Volumetric forcing. The model is to compensate the unresolved lubrication force for gaps of few grid spacings between the solid surfaces, and is validated with the examination of velocity history and fluid force acting on spherical particles falling under gravity towards a plane wall. The peak particle Reynolds number is from 7 to 58, and relative density between the fluid and the solid is up to 2.9. Excellent agreements have been observed among the simulation results, the experimental ones, and predictions from an analytical model which consists of the lubrication force proposed by Cox \& Brenner (1967), the added mass force proposed by Milne-Thomson (1968), and the history term proposed by Mei \& Adrian (1992).
\end{abstract}

Keywords: fictitious-domain, fluid force, small gaps

\section{INTRODUCTION}

Bedload sediment transport in rivers and nearcoast is fundamental in hydraulics because of its influence on the flows, and because of the resulting erosion of river banks or the seabed. However, bedload transport is extremely complicated due to dense concentration of solid phase in the highly sheared turbulent liquid which yields, and influenced by, interaction between the flow turbulence and solid particles on the bed, and among the bed particles themselves. Therefore, understanding of these phenomena is crucial to describe the bedload motion.

Aiming to numerically simulate bedload transport at feasible computational cost, our group is implementing the fictitious-domain method (Truong et al, 2005, 2006). In this method, the solid phase is treated as a fluid and governing equations are solved throughout the computational domain with fixed Cartesian grid, thus re-meshing is not required after each computation step to satisfy the non-slip condition on the surface of the particles. Rigid motions of the solid particles are constrained by adding an artificial force to the governing equations when they are solved through the solid phase, hence fluid forces acting on the solid particles are evaluated implicitly rather than integrated stress distribution over solid surfaces. This yields a fact that when gaps between the solid surfaces are less that one grid spacing, lubrication effects may not be fully solved, hence the fluid force acting on the particle is under-estimated.

In this paper, we first examine the unresolved part of the fluid force due to the lubrication effects and then propose a simple model to compensate it in the fictitious-domain simulation method, called "subgrid" correction model for lubrication force. The model is applied in tests of spherical particles approaching to a plane wall at small gaps. The results, in terms of particle velocity and fluid forces, are compared with those of Harada et al (2001), and with experimental and analytical ones.

\section{REVIEW OF THEORY}

One of the most important factors of the mechanism of inter-particle interaction is the fluid force acting on the particles when the gap between the surfaces is small compare to the particle diameters. For a sphere moving perpendicular to a plane surface, due to the squeezing effects of the fluid in the gap which satisfies the quasi-static Stokes equation, the fluid force can be described by the well-known lubrication one:

$$
F_{l}=6 \pi \mu_{f} R^{2} u_{p} / x_{g a p}
$$


in which $R$ and $u_{p}$ is the radius and velocity of the sphere, $\mu_{f}$ is the dynamic viscosity of the fluid, $x_{\text {gap }}$ is the minimum distance between the surface of the sphere and the wall. Cox and Brenner (1967) included, to lowest order in Reynolds number, the effect of fluid inertia on fluid force and proposed the equation:

$$
F_{l C}=6 \pi \mu_{f} R u_{p} \frac{1+\frac{1}{5}\left(1+\frac{R e_{p}}{2}\right) \operatorname{sln} \frac{1}{\varepsilon}}{\varepsilon}
$$

where $\varepsilon=x_{\text {gap }} / R$ and $R e_{p}=u_{p} R / v_{f}$

Their derivation, which was based on singular perturbation techniques, requires $\varepsilon<<1$ and $\varepsilon \operatorname{Re}_{p}<<1$.

When the particle is accelerating perpendicular to the wall, Milne-Thomson (1968) showed that the resulting added mass force increases by a factor of $\left[1+\frac{3}{8} \frac{R^{3}}{\left(R+x_{\text {gap }}\right)^{3}}\right]$ compared to that in unbounded domain:

$$
F_{A D}=\left[\frac{2}{3} \pi R^{3} \rho_{f} \frac{d u_{p}}{d t}\right]\left[1+\frac{3}{8} \frac{R^{3}}{\left(R+x_{\text {gap }}\right)^{3}}\right]
$$

here, $\rho_{f}$ is fluid density.

The history force acting on a sphere is expressed as the integration of a product of the acceleration of the particle and a kernel $\mathrm{K}$ :

$$
\begin{aligned}
& F_{H}(t)=6 \pi \mu_{f} R \int_{-\infty}^{t} K\left(t, s, R e_{D}\right) \dot{u}(s) d s \\
& \text { in whichRe } e_{\mathrm{D}}=\frac{\mathrm{u}_{\mathrm{p}} \mathrm{D}_{\mathrm{p}}}{v_{f}}=\frac{\mathrm{u}_{\mathrm{p}}(2 \mathrm{R})}{v_{\mathrm{f}}}, v_{f}=\mu_{f} / \rho_{\mathrm{f}}
\end{aligned}
$$

At zero Reynolds number, the kernel $\mathrm{K}$ is derived analytically and the force is known as the Basset one (Michaelides, 2003). At finite Reynolds number, the kernel $\mathrm{K}$ has been obtained mostly via numerical results (Michaelides, 2003). One such empirical expression is proposed by Mei and Adrian (1992):

$$
K(t, s) \approx\left\{\left[\frac{\pi v_{f}}{R^{2}}(t-s)\right]^{1 / 4}+\left[\frac{\pi}{2 R v_{f}}\left(\frac{u(s)}{f_{H}}\right)^{3}(t-s)^{2}\right]^{1 / 2}\right\}^{-2}
$$

$f_{H}=0.75+0.105 R e_{D}(s)$ is the correction factor for finite Reynolds number.

Based on simulation with body-fitted coordinate system, Harada et al (2001) reported fluid force acting a sphere falling toward a wall at peak Reynolds number of around 1 and 7. However, their simulation stopped at around 5\% of the particle diameter from the wall due to unstable calculations.
Following Harada et al (2001, “model B”), we will use an analytical model for the fluid force on the sphere, simply summing Eq.(1),(2), and(3). Eq.(2) is a simpler alternative to the expression they used, though equally valid. Eq.(3b) is valid for finite Reynolds number, unlike the Basset expression for $K$ employed by Harada et al(2001) . This model will be validated experimentally and numerically in section 5 for intermediate values of the gap $x_{\text {gap }}$. On that basis, we use Cox and Brenner's (1967), Eq.(1) as “ground truth” for the lubrication force.

\section{SIMULATION METHOD}

In fictitious-domain method, the same governing equations are applied over the entire flow domain using fixed Cartesian grid. The present of the body is accounted by artificial body force terms added to the momentum equations.

\section{(1) Numerical method}

The simulations were done with fictitious-domain method employing Variable-density Implicit Volumetric forcing scheme (VIV). This method has been tested extensively, as reported by Truong et al (2005, 2006). The method is summarized as follows.

Let $\Omega_{\mathrm{f}}$ and $\Omega_{\mathrm{p}}$ be the region occupied by the rigid body and the fluid, respectively, and $\Omega=\Omega_{f} \cup \Omega_{p}$ be the entire computational domain containing both the solid and fluid phases. The governing equations are the variable density Navier-Stokes equations:

$$
\begin{array}{lr}
\frac{\mathrm{D}(\rho \mathbf{u})}{\mathrm{Dt}}=\nabla \cdot \boldsymbol{\sigma}+\left(\rho_{\mathrm{p}}-\rho_{\mathrm{f}}\right) \mathbf{g}-\mathbf{f} & \text { in } \Omega \\
\nabla \cdot \mathbf{u}=0 & \text { in } \Omega
\end{array}
$$

where $\rho_{p}$ is solid density, $\mathbf{u}=(1-\alpha) \mathbf{u}_{\mathrm{f}}+\alpha \mathbf{u}_{\mathrm{p}}$ is the velocity and $\boldsymbol{\sigma}=\mathrm{p} \boldsymbol{\delta}+\mu\left[\nabla \mathbf{u}+(\nabla \mathbf{u})^{\mathrm{T}}\right]$ is the stress tensor defined over the entire domain $\Omega$ which satisfied $\left.\sigma\right|_{\Omega_{\mathrm{f}}}=\sigma_{\mathrm{f}}$. The volume fraction $\alpha$ is determined by a cosine distribution to smooth temporal and spatial changes in material properties:

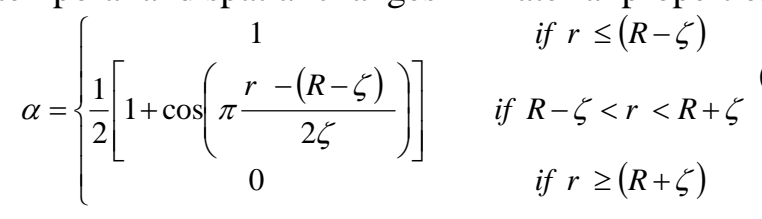

where $r$ is the distance from a grid box center to the center of sphere, and $\zeta$ is the half-width of the smoothing fringe. Truong et al (2005) reported that the results are insensitive to the choice of $\zeta$ within 1-2 grid spacings. For the simulation reported here, we chose $\zeta=1$. 


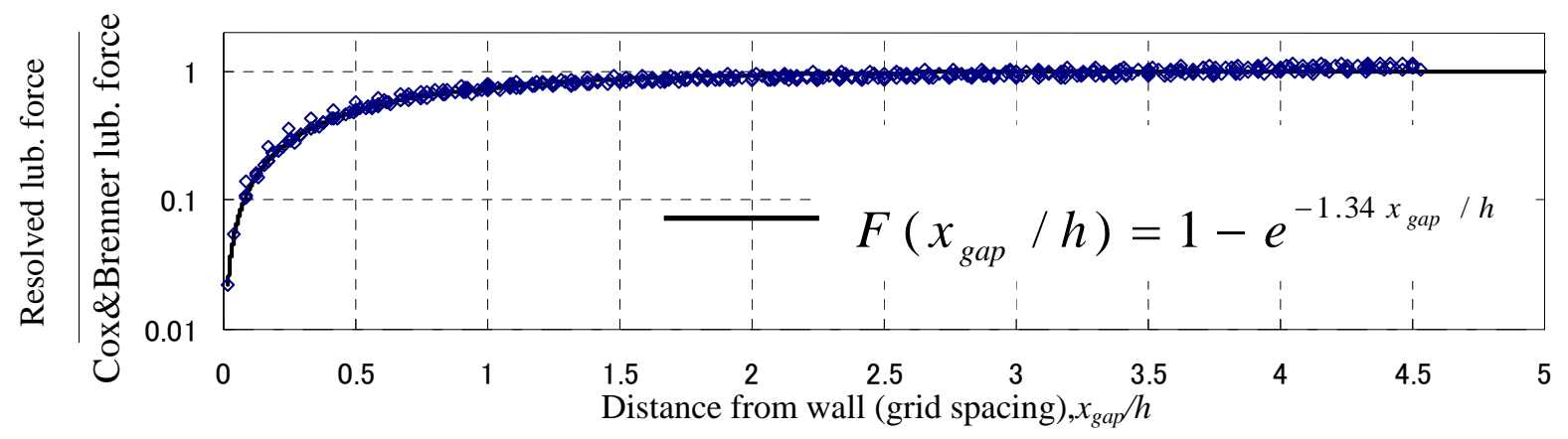

Fig.1 Ratio of "resolved lubrication force” and Cox \& Brenner’s (1967) lubrication force, Eq.(1), vs. distance from wall in grid spacing, $x_{\text {gap }} / \mathrm{h}$. Reynolds number: 10-120, number of grids per diameter: $24-70$.

The density $\rho$ and viscosity $\mu$ are determined by:

$$
\left[\begin{array}{l}
\rho \\
\mu
\end{array}\right]=(1-\alpha)\left[\begin{array}{l}
\rho_{\mathrm{f}} \\
\mu_{\mathrm{f}}
\end{array}\right]+\alpha\left[\begin{array}{l}
\rho_{\mathrm{p}} \\
\mu_{\mathrm{p}}
\end{array}\right]
$$

where $\mu_{p}$ is the viscosity employed for the solid phase, which is usually set proportional to density $\mu_{p}=\mu_{f} \frac{\rho_{p}}{\rho_{f}}$ the virtual viscosity to avoid constraining the time step any more than necessary.

f is the artificial body force to recover rigid motion. In the VIV method, the particle physical density is used in the momentum equation, and consequently $\mathbf{f}$ causes the immersed solid particle to move with a physically correct acceleration. The particle tentative velocity is treated as unknown and be solved together with the fluid motion. The rigidity constraint force density at each grid cell in the computational domain is then defined by:

$$
\mathbf{f}=\left\{\begin{array}{c}
-\frac{\mathrm{D}\left(\rho_{\mathrm{p}} \mathbf{u}_{\mathrm{p}}\right)}{\mathrm{Dt}}+\nabla \cdot \boldsymbol{\sigma}+\left(\rho_{\mathrm{p}}-\rho_{\mathrm{f}}\right) \mathbf{g} \text { in } \Omega_{\mathrm{p}} \\
0 \quad \text { in } \Omega_{\mathrm{f}}
\end{array}\right.
$$

or extend to the entire domain $\Omega$ as

$$
\mathbf{f}=\alpha\left[-\frac{\mathrm{D}(\rho \mathbf{u})}{\mathrm{Dt}}+\nabla . \boldsymbol{\sigma}+\left(\rho-\rho_{\mathrm{f}}\right) \mathbf{g}\right] \text { in } \Omega
$$

$\mathrm{D} \mathbf{u}_{\mathrm{p}} / \mathrm{Dt}$ is determined to satisfy rigid motion, and so that $\mathbf{f}$ yields zero net injection of momentum within each particle's fictitious domain, and the net momentum added to the fluid is zero. Thus the rigid motion of any immersed particles at any instant could be recovered from the corresponded velocity field.

\section{(2) Correction model for "subgrid" lubrication force}

Davis et al (1986) assumed that the lubrication force dominates from a gap of $0.5 \%$ of the sphere diameter in their simulation of elastohydrodynamic collision of two spheres. Adopting such assumption,
Table 1 Tested particle Reynolds numbers. $h$ is "threshold” grid spacing below which curve of Fig.1 does not change.

\begin{tabular}{|c|c|c|}
\hline $\operatorname{Re}$ & $\frac{D_{p}}{h}$ & $\left.\operatorname{Re}\right|_{1 \mathrm{~h}}=\frac{U_{o} h}{v}$ \\
\hline 10 & 24 & 0.42 \\
\hline 20 & 30 & 0.66 \\
\hline 30 & 36 & 0.83 \\
\hline 60 & 40 & 1.5 \\
\hline 80 & 60 & 1.33 \\
\hline 100 & 66 & 1.52 \\
\hline 120 & 70 & 1.71 \\
\hline
\end{tabular}

in our simulation, it requires the number of grid points per diameter, $D_{p} / h$, to be 200 or more. This is prohibitively dense, especially for simulation of hundreds or thousands of particles. Consequently, a model to recover the un-resolved lubrication effects for coarser grid resolution is required.

Cox and Brenner's (1967) lubrication force (Eq.(1)) is used as a benchmark to evaluate the unresolved lubrication force in our simulation. Hydrodynamics acting on a sphere with neutral density approaching to a rigid wall from a gap of $10 \%$ to $20 \%$ of the diameter with a constant velocity $\mathrm{U}_{\mathrm{o}} \quad$ corresponding to Reynolds number, $R e_{D}=U_{o} D_{p} / v_{f}$, from 10 to 120 were evaluated. The 'resolved lubrication force' is compared to Cox and Brenner's (1967) equation and shown in Fig.1 as a function of number of grid spacing from the wall. Note initial position of the particle does not change the result.

For each Reynolds number, the simulation was run with many grid resolutions, i.e. many numbers of $D_{p} / h$, and it is found that at a certain Reynolds number, there was a number of $D_{p} / h$ above which the ratio of $\frac{\text { 'resolved lubrication force' }}{\text { Cox \& Brenner's lubrication force }}$ of all Reynolds number in the tested range fell into a single curve, as presented in Table 1 . Note that at these values of the 'threshold $D_{p} / h$ ', all the Reynolds 


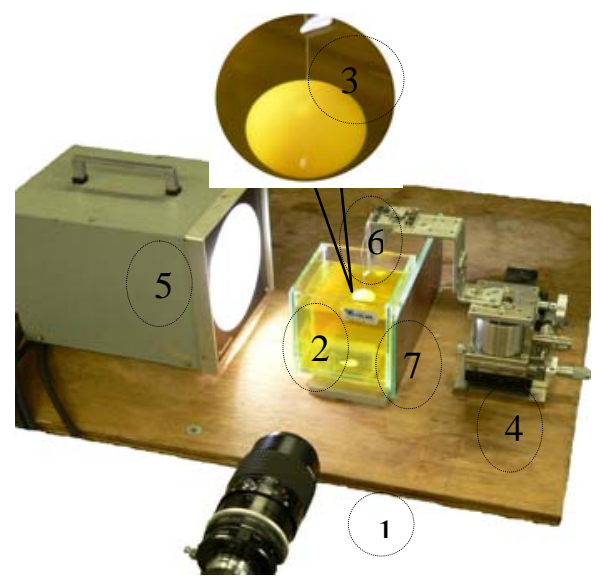

Fig.2 Experiment apparatus: 1) camera, 2) glass tank $\left(10 \times 10 \times 10 \mathrm{~cm}^{3}\right)$ filled with machine oil, 3) sphere, 4) stage, 5) light source, 6) tweezers, 7) mirror.

Table 2 Experiment and simulation conditions.

\begin{tabular}{|c|c|c|c|c|}
\hline & \multicolumn{2}{|c|}{ Nylon sphere } & \multicolumn{2}{|c|}{ Glass sphere } \\
\hline & Test P1 & Test $P 2$ & Test G1 & Test G2 \\
\hline$\rho_{p}\left(\mathrm{~g} / \mathrm{cm}^{3}\right)$ & 1.129 & 1.356 & \multicolumn{2}{|c|}{2.525} \\
\hline$D_{p}(\mathrm{~cm})$ & 2.27 & 1.71 & \multicolumn{2}{|c|}{2.45} \\
\hline$\rho_{f}\left(\mathrm{~g} / \mathrm{cm}^{3}\right)$ & 0.869 & 0.873 & 0.877 & 0.869 \\
\hline$\mu_{f}(\mathrm{~g} / \mathrm{cm} . \mathrm{s})$ & 0.839 & 0.219 & 2.17 & 0.839 \\
\hline$x_{\text {gap-initial }} / D_{p}$ & 0.1 & 0.152 & \multicolumn{2}{|c|}{0.164} \\
\hline$U_{t}(\mathrm{~cm} / \mathrm{s})$ & 24.7 & 39.2 & 66.7 & 91.8 \\
\hline$\rho_{p} / \rho_{f}$ & 1.3 & 1.55 & 2.88 & 2.91 \\
\hline
\end{tabular}

numbers based on gap $\left.R e\right|_{1 \mathrm{~h}}$ are less than 2, where Cox and Brenner's (1967) expression for lubrication force should be applicable.

This curve is fitted by an exponential function:

$$
\frac{\text { 'resolved lubrication force' }}{\text { Cox \& Brenner's lubrication force }}=1-e^{-1.34 x_{\text {gap }} / h}
$$

in which $x_{\text {gap }} / h$ is the distance from the wall, in grid spacing. Therefore the added force required to compensate the lubrication effects is:

$$
f(x)=e^{-1.34 x_{\text {gap }} / h}
$$

with the condition that $\left.\operatorname{Re}\right|_{1 \mathrm{~h}}$ is less than 2. This extra force is accounted to the constraint of the rigid motion of the particle after each computational time step.

\section{EXPERIMENT}

The experimental conditions are presented in Table 2. Nylon and glass spheres were positioned at specific distances above a bottom wall of a glass tank which is filled with mixtures of machine oils. A nylon fishing string with a diameter of less than $0.2 \mathrm{~mm}$ is glued on the top of the sphere and attached to a tweezers which could be moved in the vertical direction by a stage to a resolution of $5 \mu \mathrm{m}$. The light source was a strobe or a light bulb opposite to a mirror so that the particle was illuminated from both directions. To prevent the fluid from warming up, the light source was turn off right after each run. A camera running at $240 \mathrm{~Hz}$ with a $105 \mathrm{~mm}$ micro-lens was connected to a computer to provide magnified view for adjusting the position of the particle and to record images of the particle's motion. To drop the sphere, the tweezers were released when the fluid inside the tank was observed to be at rest. The velocity of the particle at a vertical position $\left(x_{i}+x_{i+1}\right) / 2$ above the wall was determined by $u_{p}=\frac{\left|x_{i}-x_{i+1}\right|}{\Delta t}$, where $\Delta t=(1 / 240)$ second, $x_{i}$ and $x_{i+1}$ are the particle's positions in two successive images.

Viscosities of the machine oils were determined by dropping sphere method. Terminal velocities of small plastic spheres in the fluids were measured and then the drag coefficients were obtained. Terminal Reynolds numbers, and hence corresponding viscosities, were calculated from the standard drag curves (Clift et al, 1978). These standard curves, via iteration process, also help to determine the terminal velocities of the test spheres in corresponding experimental conditions as shown in Table 2.

\section{RESULTS}

(1) Comparison with Harada et al's (2001) result

In Fig. 3, our simulation results are plotted together with Harada et al's (2001) experimental and simulation data for their test Case B of which the peak Reynolds number is about 7 , relative density is 1.144 , and a domain of (3Dp) ${ }^{3}$. Excellent agreement can be seen. With our fictitious-domain simulation method, the simulation stops only when proceeding to the next time step, the physical particle surface is out of the computational domain. The convergence of the simulation results with increasing grid resolution is confirmed to have been achieved for 24 and 32 grid points per diameter, though not 12. The significance of the lubrication force correction (Eq.(11)) at a few grid points from the wall is clear with the test of $24 \mathrm{grid} / \mathrm{Dp}$. Harada et al's simulation results lie closer to the trajectory calculated with the correction included, but their two experimental points nearest the wall lie closer to the uncorrected trajectory. We return to this issue in the next subsection.

Also shown in this figure is our analytical model for the fluid force acting on the particle near wall, 

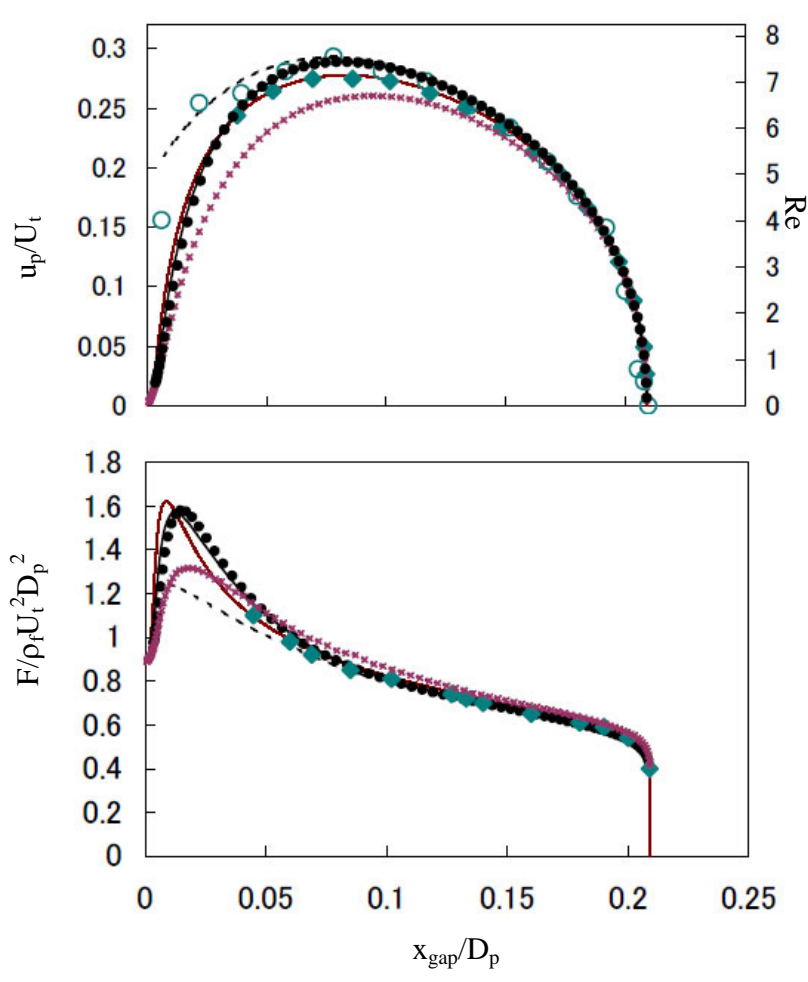

Fig.3 Comparison to Harada et al (2001), test Case B; Our simulation: 32grid/Dp, 24grid/Dp, - _ - 24grid/Dp: no lubrication force correction, - - =x= -12grid/Dp; OHarada et al's (2001) experiment; Harada et al's (2001) simulation; __ our model

$\mathrm{F}=\mathrm{F}_{\mathrm{IC}}+\mathrm{F}_{\mathrm{AD}}+\mathrm{F}_{\mathrm{H}}$. From the initial conditions, velocity of the particle is integrated from the fluid force model, and again the fluid force at each position is evaluated simultaneously from the velocity history and the instantaneous one. This model is in good agreement with both our simulation and Harada et al's (2001) results.

\section{(2) Comparison to Experiments}

In Fig. 4 and Fig. 5, results from the simulation and from the fluid force model are presented together with the experimental data from tests with the plastic and glass spheres, respectively.

Consider first the values of gap for which experimental data are available. The simulation results, which in all cases have been checked to converge with increasing resolution, agree extremely well with the force model, and consistently pass through the center of the experimental scatter. Over the tested range of relative density and Reynolds number, this provides strong evidence that both the simulation method and the force model are accurate to within a few percent for these "intermediate" values of gap.

Next, we consider the validity of the lubrication force correction used in our simulations, which becomes significant when the gap is less than about one grid point (Fig. 1), over the range of parameters tested. Experimental data for such small gaps is only available in the test of Harada et al (Fig. 3), and our test P1 (Fig 4), for which the peak Reynolds number of 10 , and relative density of 1.3 are very similar to Harada et al. As already remarked, the former data do not provide support for the lubrication correction. By contrast, our test P1, with substantially more data points, does indeed agree well with the simulation results that include the lubrication correction.

Though the direct experimental support for our lubrication correction is thus only partial, we argue that the analytical model can serve as a credible benchmark at the small values of $x_{\text {gap }}$ now under consideration. First, consider the validity of the added mass contribution (Eq.(2)) during the final approach. Physically, its value does not change more than a few percent. Numerically, we expect the velocity profile in the gap is "smeared" out somewhat, but we confidently expect that smearing of the flow in the gap should not pollute this component of wall-normal momentum in the resolved flow, and conclude that the added-mass effect does not induce errors in the fluid force on the simulated particle.

We have no argument for the continued theoretical validity of the history term (3) at small gaps, nor for the correct treatment of this effect in the simulations when the gap becomes small. However, the relevant curves in Figs 4 and 5 show that the relative importance of this term decreases as particle Reynolds number increases.

Finally, there seems little doubt that the lubrication contribution, Eq.(1), becomes increasingly accurate as the gap decreases. Thus, together with the preceding arguments about the contributions (2) and (3), we believe that the analytical model, validated by nearly all of the available experimental data, should be at least equally valid for the small gaps not covered by the measurements, and thus can serve as a benchmark for the numerical simulations.

Now it is possible that the non-neutral densities in the simulation runs corresponding to our experimental cases could induce an error in the lubrication flow. But with the preceding justification, we now invoke our analytical model as a benchmark. The good agreement between this model and the simulations then suggests that the latter, including the lubrication correction, are indeed accurate.

Deceleration of the particles due to the presence of the wall can be seen in all tests. The particles moving with smaller Reynolds numbers are, as expected, affected by the wall at larger gaps. 

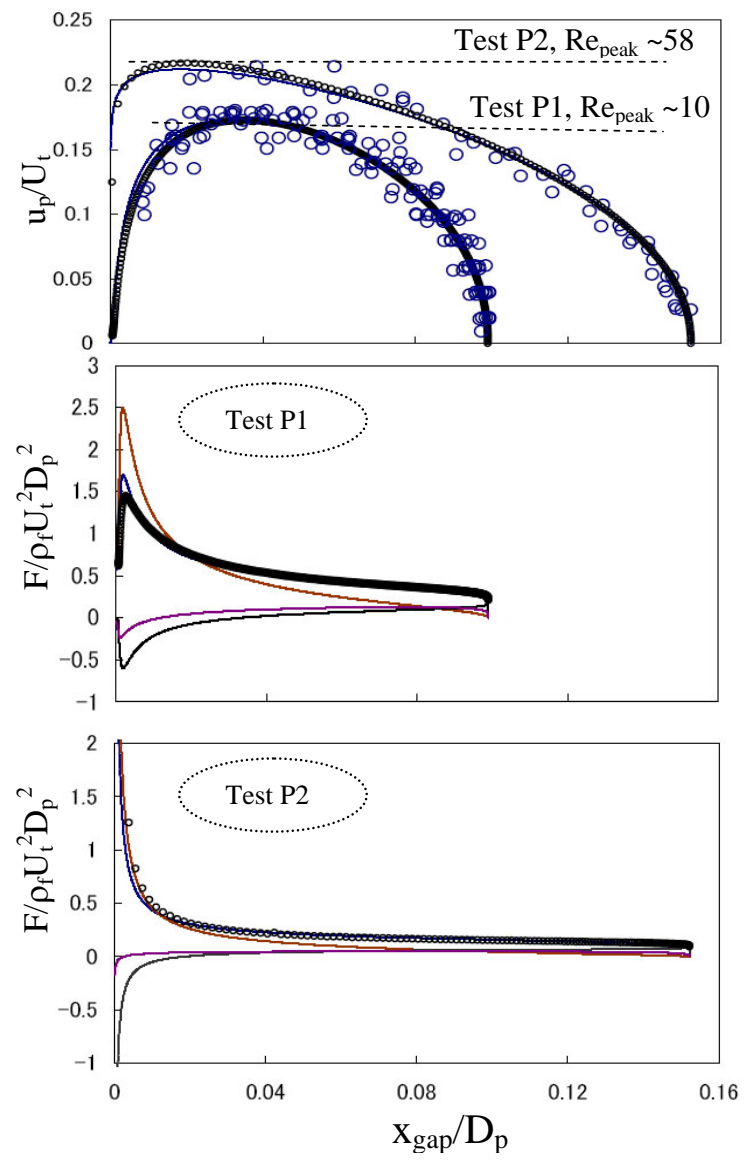

Fig.4 Tests with plastic sphere in machine oils (symbols as in Fig. 5)

At the beginning of motion, the two unsteady forces, the added mass force $F_{A D}$ and the history force $F_{H}$, are dominant over the lubrication one, $F_{I C}$. Near to the wall, the deceleration of the particle causes $F_{A D}$ and $F_{H}$ to change their sign, $F_{I C}$ increases and becomes dominant. It is noticed that at very small gap, $F_{H}$ is negligible compared to $F_{I C}$ while the $F_{A D}$ is not. Therefore, a distance from wall where the other forces can be completely neglected compared to the lubrication force is hard to specify here.

\section{SUMMARY}

Overall agreements in velocity history and fluid force of the spheres falling onto a plane surface have been observed among the simulation results, the analytical, and the experimental ones. The model of "subgrid" lubrication force added to the fictitiousdomain VIV method to compensate the unresolved lubrication force is proved to work very well in the tested range of peak Reynolds number from 7 to 58, and relative density up to 2.9. This result makes us optimistic for applying this model to the fictitious domain simulation of dense-phase granular flow in liquid. In the near future, tests with higher Reynolds numbers, and accelerometers to monitor particle trajectories, are planned.

ACKNOWLEDGEMENT: We are grateful for support from the Monbukagakusho Ministry for a scholarship for Mr. Nguyen.

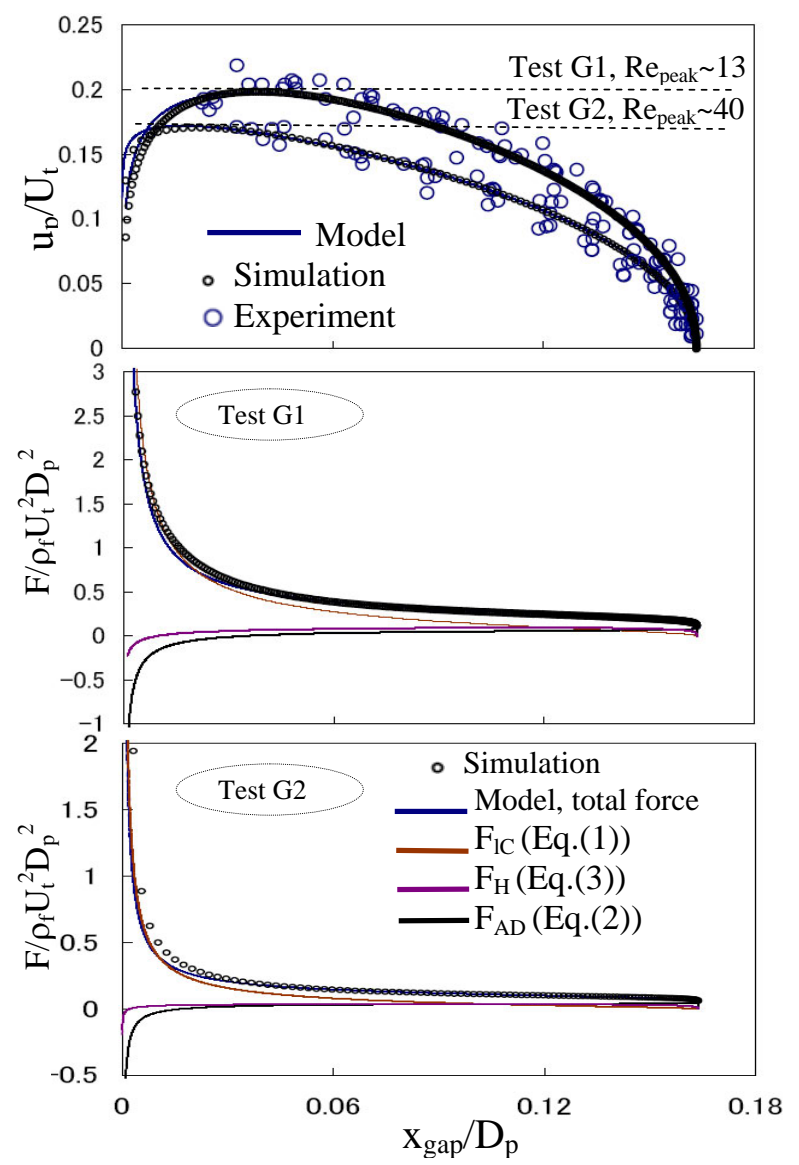

Fig. 5 Tests with glass sphere in machine oils

\section{REFERENCES}

1. Clift, R., Grace, J.R., Weber. M.E.: Bubbles, Drops, and Particles, Academic Press, New York 1978.

2. Cox, R.G., Brenner, H.: The slow motion of a sphere through a viscous fluid towards a plane surface-II Small gap widths, including inertial effects, Chemical Engineering Science, Vol. 22, 1753-1777, 1967.

3. Davis, R.H., Serayssol, J.M., Hinch E.J.: The elastohydrodynamic collision of two spheres, Journal of Fluid Mechanics, Vol. 163, 479-497, 1986.

4. Harada, S., Tanaka, T., Tsuji, Y.: Fluid force acting on a falling particle towards a wall, JSME International Journal, Serie B, Vol. 44, No 4, 520-525, 2001

5. Mei, R.W., Adrian, R.J.: Flow past sphere with an oscillation in the free-stream velocity and unsteady drag at finite Reynolds-number, Journal of Fluid Mechanics, Vol. 237, 323-341, 1992.

6. Michaelides, E.E.: Hydrodynamic force and heat/mass transfer from particles, bubbles, and drops - The Freeman scholar lecture, Journal of Fluids EngineeringTransactions of the ASME, Vol. 125, issue 2, 209-238, 2003.

7. Milne-Thomson, L.M.: Theoretical Hydrodynamics, Macmillan Education, London, 1968.

8. Truong, H.V., Wells, J.C., Tryggvason, G.: Explicit vs. implicit particle-liquid coupling in fixed-grid computations at moderate particle Reynolds number, FEDSM2005-77206, ASME FED Summer Meeting, June 19-23, 2005, USA.

9. Truong, H., Wells, J., Nguyen, A.: An experimental check on particle dynamics simulation of erodible particle beds; the rotating tumbler, Annual Journal of Hydraulic Engineering, JSCE, Vol. 50, 127-32, 2006.

(Received September 30, 2006) 